\title{
EVALUTION AND CALIBRATION OF MINIATURE CIRCUIT BREAKER
}

\author{
Ramandeep Singh (1), Baljinder Singh (2) \\ Research Scholar (1) \\ raman_sandhuin@yahoo.com \\ Asst. Prof (2), GTBKIET Chhapianwali, Malout \\ bsinghgtb@gmail.com
}

\begin{abstract}
The purpose of this paper is to examine the significant developments in MV and LV switchgear technology over the last few decades and why they are only recently beginning to benefit the users. A recent development now has sufficient field experience to show how through innovative design it has been possible to vastly improve an auto-reclosing circuit breaker. The author believes that the same innovative ideas will soon be applied to all types of switchgear. The next generation of military switchgear is likely to be developed for the Electric Warship. Irrespective of which distribution supply is used the next generation of switchgear should benefit from the new technology. Calibration of Miniature Circuit Breaker takes a lot of time. Calibration is done to satisfy the International Standards so that all the MCBs are standardized. In this paper we concerned with decreasing this calibration time of MCBs whose rated current is less than or equal to 32 Amps. Calibration can be done by two methods, wiz, and Dual current and single current parameter method. This paper also deals with the decrease in the Calibration time and increase in Production efficiency by the use of later method which is a modification of the former one proposed and implemented here. This is done by employing faster motor for the calibration. A recent development now has sufficient field experience to show how through innovative design it has been possible to vastly improve an auto-reclosing circuit breaker. The author believes that the same innovative ideas will soon be applied to all types of switchgear.
\end{abstract}

\section{INTRODUCTION}

The main application of switchgear is in the protection of circuits against damage caused by faults and the restoration or preservation of supplies to as large a part of the system as possible following a fault. In this function they must add reliability to the system rather than contribute to system problems. Providing fault occurrences are rare then system disruption caused by the switchgear, either for maintenance or operational problems has to be very rare. Hence the main objective of any switchgear development must be to improve the reliability of the switchgear itself as well as its performance as a protective device. MCB is a protective device which operates automatically on the fault and the supply can be resumed by near switching action after clearance of the fault. A Miniature Circuit Breaker is a electromagnet protective device which is used for feeding electricity to houses, railways, industries, etc. The main advantage of using this device is the protection which it provides against short-circuit and overload condition. Unlike a fuse, which operates once and then has to be replaced, a circuit breaker can be reset (either manually or automatically) to resume normal operation. Circuit breakers are made in varying sizes, from small devices that protect an individual household appliance up to large switchgear designed to protect high voltage circuits feeding an entire city

\section{0's SWITCHGEAR}

Oil and air circuit breakers dominate LV and MV switchgear markets.

\section{- Air circuit breakers}

Air has been used as the insulator in switchgear across the entire voltage range from miniature circuit breakers at domestic voltage to air-blast circuit breakers at transmission voltages up to $800 \mathrm{kV}$. For the purpose of this paper the term air circuit breaker will be used to describe a three-phase circuit breaker using air at atmospheric pressure as the insulator.

Early switches were plain-break which stretched an arc between a stationary and moving contact with no means of arc control. Arcing times were consequently long and voltage and current ratings limited. Performance was increased by the use of various arc control devices, probably the best and therefore the most commonly used arc control device is the arc chute.

Arc chutes comprise a number of bare metal plates arranged at right angles to the length of the arc chute with spacers between the plates to allow the arc to be split up into a number of series smaller arcs, increasing its resistance and extracting heat. Eventually the arc is no longer able to sustain itself. When interrupting high short circuit currents the anode to cathode voltage drop is approximately $30 \mathrm{~V}$ across each pair of plates. In dc circuit breakers if enough plates are used in series the arc voltage across the chute can be greater than the system voltage, forcing the current down to zero and thereby interrupting the current. 


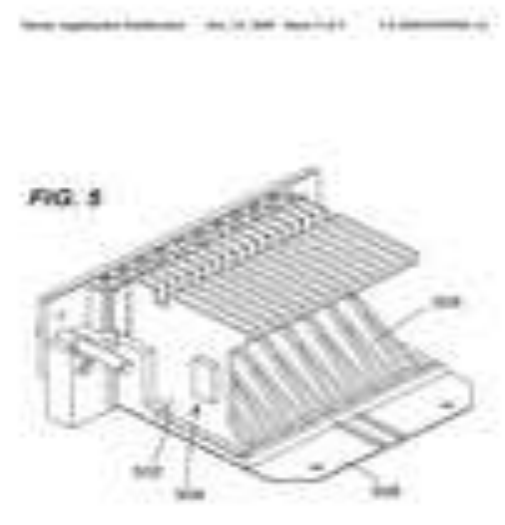

Fig 1(a) Cross-section through arc chute

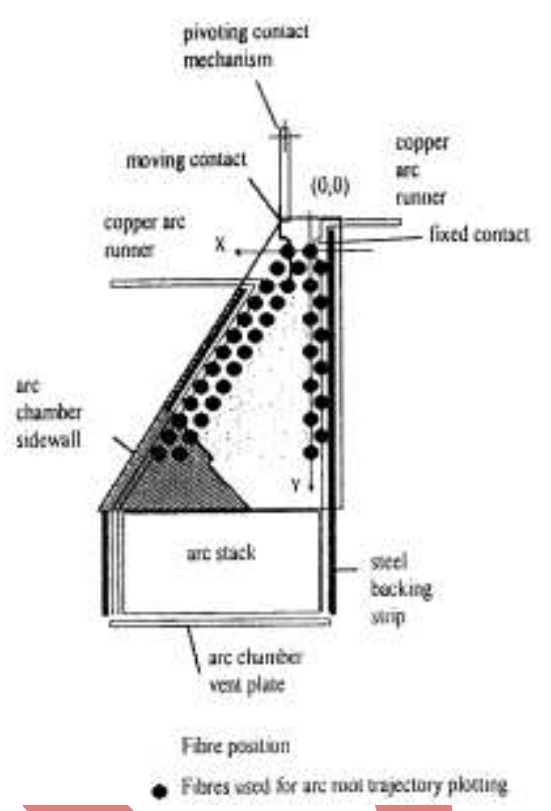

Fig1 (b) Electro-magnetic forces on arc

Fig 1(a) shows the arc in its final position in a typical arc chute. The arc is initiated at the point of contact separation between the fixed and moving contacts and is transferred into the chute by electromagnetic and thermal forces causing the arc to run away from the contacts along the arc runners. Arcing times are dependent upon current and voltage and can be relatively long.

In order to withstand the transient recovery voltage at the instant of interruption of an inductive current and also any tran sient overvoltages the contact separation in air is large. The same electromagnetic forces, which move the arc into the chute at high currents, also act on the contacts attempting to blow them apart. Large contact pressure springs are used to counter the forces.

The combination of the long contact travel and the compression of heavy contact pressure and opening springs during the closing stroke necessitated a powerful operating mechanism.

\section{0's SWITCHGEAR \\ Firstly vacuum and later rotating arc SF6 circuit breaker}

\section{- Vacuum circuit breakers}

Vacuum interrupters became commercially available in the early 1970's. The vacuum bottle is sealed for life and cannot be maintained and is capable of more fault interruptions than would be seen during the life of the switchgear without wearing out. The dielectric strength of the high vacuum is such that a contact travel of only $6 \mathrm{~mm}$ can be used at a rated voltage of $12 \mathrm{kV}$.

The advantages of the new technology was immediately obvious to several manufacturers and signalled the end of any further development in oil or air circuit breaker technology at medium voltage. The cost of the early vacuum interrupters was substantially more than comparably rated oil or air interrupters, which is probably why they do not have a significant usage even today at low voltage.

To off-set the additional cost of the vacuum interrupters to compete with oil circuit breakers three UK manufacturers produced fixed vacuum circuit breaker designs in order to reduce complexity, costs and size. The fixed design was made possible by the maintenancefree interrupters. However, the operating mechanism still demanded lubrication, occasional checks and resetting. Being too complex to rely upon for life it had to be housed in a separate low voltage compartment, connected to the three vacuum interrupters by a number of operating shafts, links, pivot points and insulators.

The first fixed designs failed to gain universal approval, mainly due to the leap of faith required in accepting manufacturer's claims that vacuum interrupters would prove to be truly maintenance free and would not lose their vacuum integrity after a period of time. Eastern Electricity who installed a large number of this type of switchgear from 1973 into primary substations claims that their operational experience has been trouble free. 


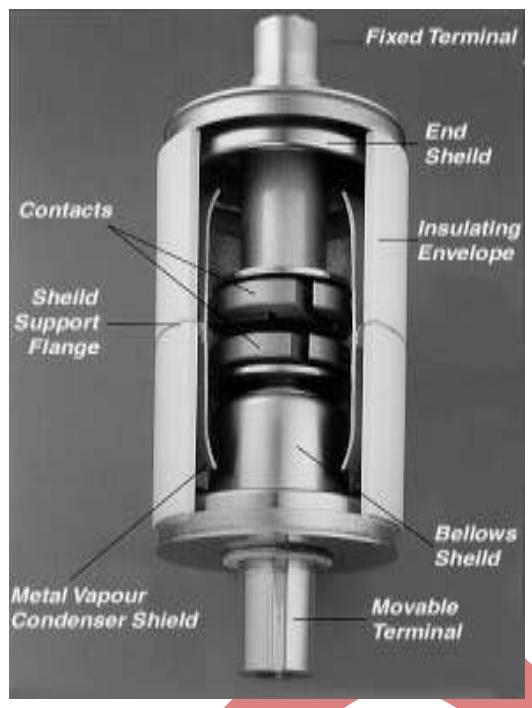

Fig 2 Typical Vacuum Interrupter

\section{- Rotating arc SF6 circuit breakers}

This technology was developed by South Wales Switchgear. The rotating arc interrupter is as inexpensive as oil or air arc control devices with almost the reliability and performance of a vacuum interrupter. SF6 is a highly insulating, heavy, non-toxic gas which decomposes during the arcing process but very quickly recombines at current zero regaining its dielectric properties. Fault currents up to $20 \mathrm{kA}$ can be cleared at $12 \mathrm{kV}$ with a $50 \mathrm{~mm}$ gap (approx) at a gas pressure of a 2.0 bar. The arc current is transferred during contact separation through a solenoid of a few turns of copper strip generating a magnetic field causing the arc to rotate around the inner ring of the solenoid. The rapid movement of the arc through the SF6 gas causes cooling and enables extinction at the first current zero at high fault currents.

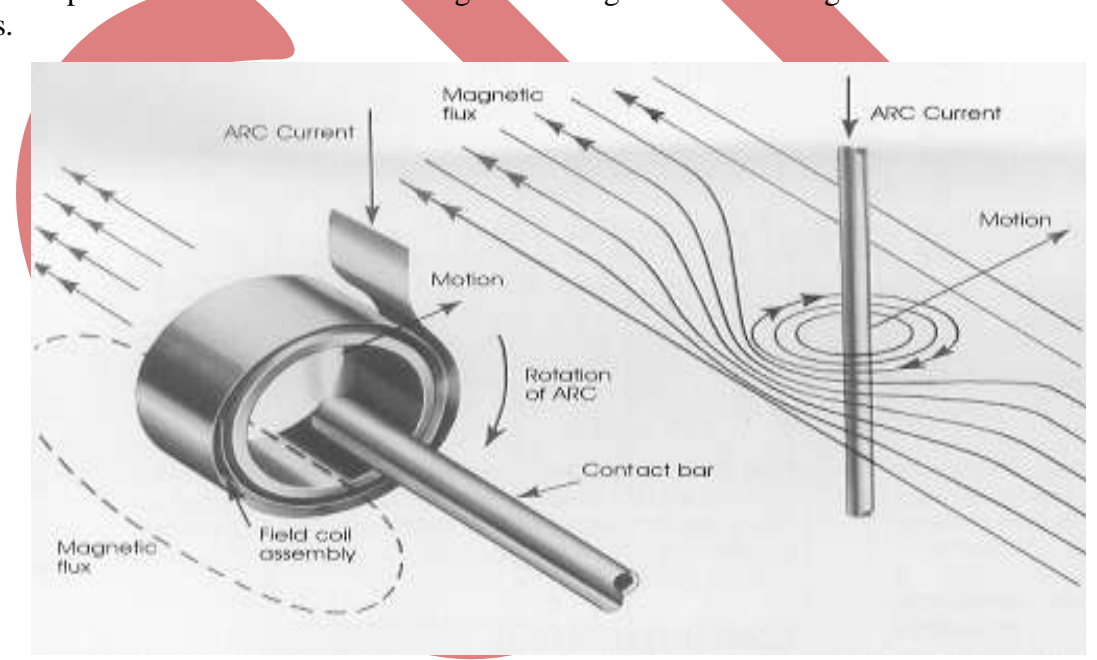

Fig 3 Rotating arc principal

\section{0's SWITCHGEAR}

No new interrupting devices have become commercially available in MV or LV since the advent of vacuum interrupters and rotating arc SF6. During the 1980's the main thrust of development activity was to provide better, more cost-effective circuit breakers using the new technologies. Vacuum interrupters have become smaller for a given rating and less expensive. Nearly all new circuit breakers developed during this period were withdrawable designs.

To reduce the size of substation batteries and chargers that provided the power source for opening and tripping of the circuit breakers, motor-wound spring mechanisms became standard equipment on all medium voltage and low voltage circuit breakers. A spring is charged by an electric motor drawing a few amps over a period of a few seconds in place of the earlier closing solenoid that took a current of many tens of amps for a fraction of a second. The spring energy is released by a smaller solenoid releasing a latch. As the spring relaxes it drives the mechanism components, compressing the contact pressure and opening springs and forcing the moving and fixed contacts of the interrupter together.

Whilst the substation battery (until the widespread use of electronic protection relays) could be reduced in size the mechanisms became even more complex compared to solenoid mechanisms. Despite claims from some manufacturers users were experiencing an increase in switchgear problems. 


\section{0's SWITCHGEAR}

Vacuum circuit breakers have emerged during this decade as by far the most preferred technology for primary substation medium voltage switchgear as field service experience shows almost zero failure rate of vacuum interrupters and interrupter prices fall.

The fixed switchgear concept has made a comeback with SF6 insulated vacuum switchgear and air insulated SF6 switchgear with conventional mechanisms in the low voltage compartment driving the interrupters through linkages.

The most significant advance in switchgear technology in many years has come with the introduction of the GVR pole mounted autorecloser which included a magnetic actuator mechanism and other radical innovations which are presently shaping new developments in medium voltage switchgear. The operating mechanism is now as reliable as the interrupter.

\section{THE 2000's}

New designs of switchgear will evolve, designed from scratch with every component as simple and reliable as possible to capitalise on the potential of the magnetic actuator mechanism to be as trouble free as the latest interrupter technology. This concept should be applied to low voltage switchgear, finally replacing the old technology air circuit breakers at this voltage and perhaps later to EHV switchgear.

Medium voltage switchgear of such a design is the Eclipse (below)

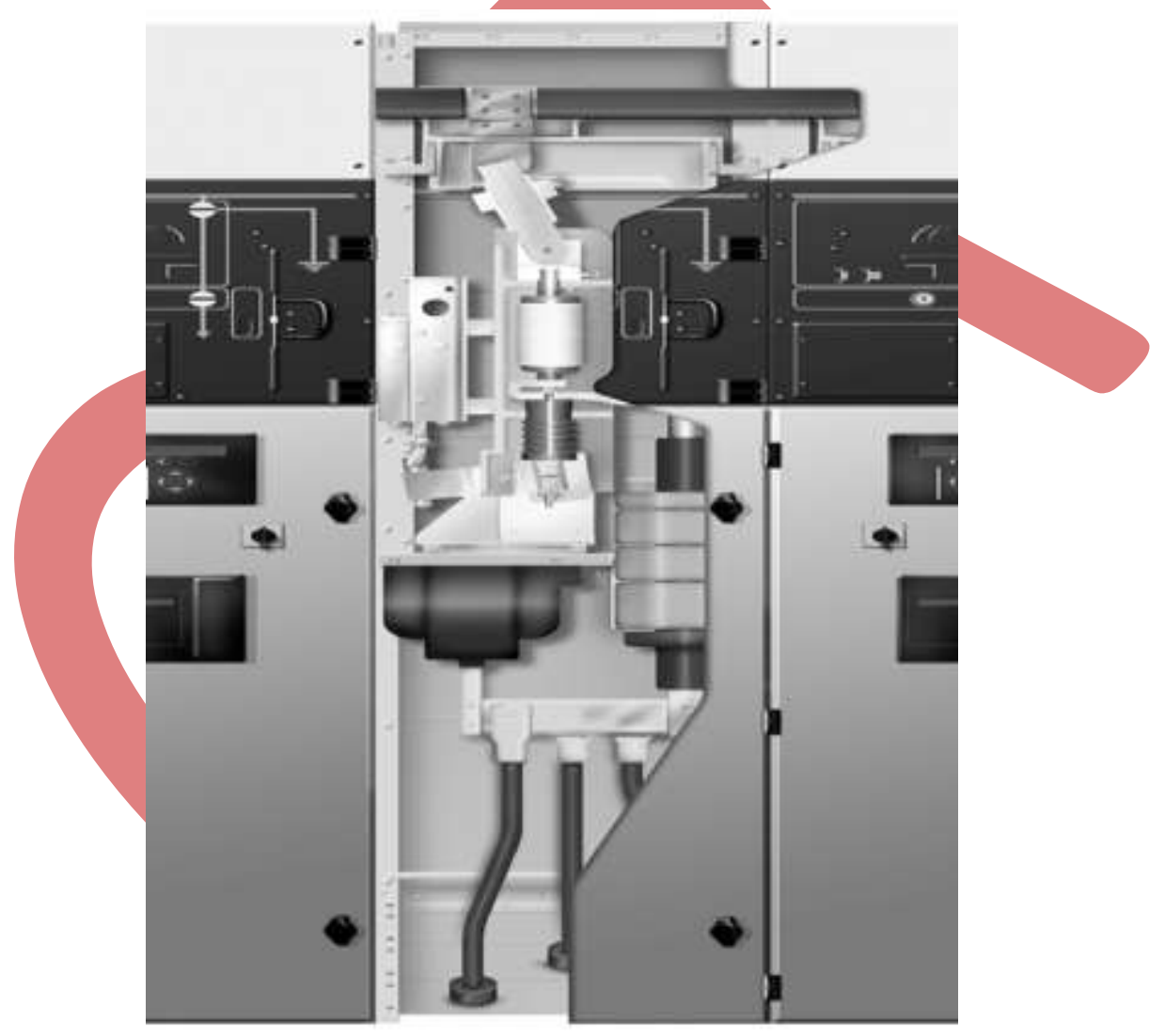

Fig 4 Eclipse - fixed vacuum air insulated switchgear with magnetic actuator drive

\section{(a) Working Principle in Short Circuit Condition:}

According to Faraday's, when current is passed through a solenoid, flux is produced around the coil, due to this the medium inside the coil becomes magnetized \& there is a force of attraction between the 2 parts (Anchor assembly \& Coil rivet) only if it is a magnetic material .By this, plunger trips the mechanism.

\section{(b) Working Principle of Bimetal For Protection From Overload:}

The Bimetal works on the cantilever principle. The different elements have different thermal expansion. When 2 different elements were bonded together physically with different thermal expansions \& exposed to high temperatures it turns into curvature. When we fix one end of the Bimetal then all the deflection happens at the free end, this is called Cantilever principle 


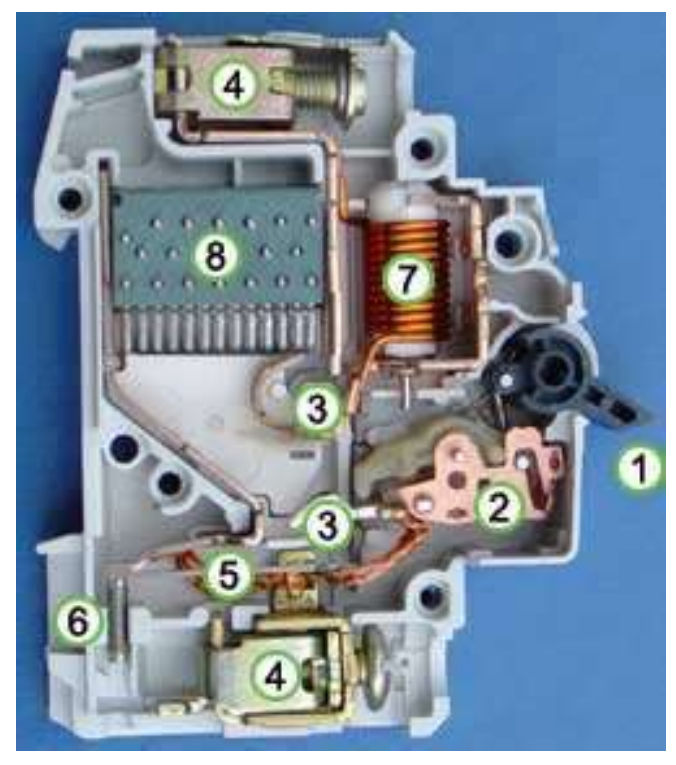

Fig:-5 Important parts of an MCB

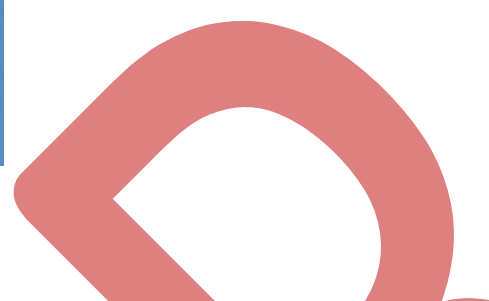

Some of the important parts of an MCB are :

1. Actuator lever

\section{Actuator mechanism}

\section{Bimetallic strip}

6. Calibration screw

\section{Solenoid}

\section{Arc divider/extinguisher}

\section{CALIBRATION}

After the whole assembly of the MCB the most important step comes in i.e. Calibration. In general use, calibration is often regarded as including the process of adjusting the output or indication on a measurement instrument to agree with value of the applied standard, within a specified accuracy. Calibration makes the whole lot of MCBs to be uniform. This process is followed by the verification step, after which they are sent for packaging.

\section{A. THE CALIBRATION PROCESS}

In the production process of MCB, the position of the Grub screw of each MCB is arbitrary. It is not set accordingly, because of which we try to achieve the tripping time of every MCB approximately same according to the standards set by the BIS(Bureau of Indian Standards). In order to make the tripping time same for each MCB , for the same rated current, the grub screw of each MCB is positioned in such a way that the deflection of bimetal is same and all the MCB's trip accordingly. So the gap between the bimetal and the plunger is made uniform for all the MCBs. For this calibration process, there is a bench where 10 MCB's are put simultaneously and calibration i.e. adjustment of the grub screw is carried out. This is done by making the MCB trip at the rated current in the specified time, by using motors to rotate the grub screw.

Maximum deflection required for calibration $=2.55 \mathrm{~mm}$

Pitch of the Grub screw $=0.55 \mathrm{~mm}$ (given)

(i.e. for every rotation the screw moves by $0.55 \mathrm{~mm}$ towards the plunger.)

$0.55 \mathrm{~mm}$ movement requires ::: 1 rotation

$2.5 \mathrm{~mm}$ movement will require ::: $(1 / 0.55) * 2.5=4.54$ rotations (for $16 \mathrm{~A} \mathrm{MCB}$ )

\section{B. Dual Current Parameter Method}

The Dual Current Parameter Method, is divided in four time intervals. Initially, we give a current which is very less as compared to the rated current, for time T1 and this time T1 is almost equal to 1sec. This is known as Justification Time. This process is solely for the purpose of continuity test of the circuit (MCB). After this, we linearly increase the current till it reaches to 2.55 times the rated current. 
This current is denoted by I1. This current I1 is given till the time equal to T2 and this current and time is enough to provide the maximum deflection to the Bi-metal, which is equal to $2 \mathrm{~mm}$. But, At the same time, it should not touch the Anchor, or else it will cause the MCB to trip. This time is known as Preheating Time and it helps to make the bi-metal flexible enough to carry on the further processes. This time varies from MCB ratings and it can minimum be 12 seconds and maximum be 25 seconds. After this a Stabilizing current(denoted by I2), which is between $1.13 \mathrm{In}$ to $1.45 \mathrm{In}$, generally taken to be $1.3 \mathrm{In}$, is given to the MCB. This stabilizes the Bimetal to a medium position, giving a deflection of around $1 \mathrm{~mm}$ (average). This time is different for each rating of MCB and is denoted by T3, known as Stabilizing Time. After carrying out the three processes given above, Calibration is carried out. This is done at a stable current $\mathrm{I} 2$. For the calibration, we use a $15 \mathrm{rpm}$ motor which rotates the grub screw. It rotates the screw till the time the MCB does not trip, and the time taken is also noted down, known as Calibration Time. Since motor is of 15 rpm, we generally require around 4 revolutions to cause a movement of the screw grub by $2.5 \mathrm{~mm}$. Therefore the total time required for the whole process is around $1 \mathrm{~min}$ (for 16Amp rating)

\section{Single Current Parameter Method}

The Single Current Parameter Method comprises of just three time intervals and is done solely purpose of decreasing the production time and increasing the production of the MCB's in the beginning, the same justification current is given for time T1, equal to 1sec. After this, a current equal to $2.55 \mathrm{In}$ is given for preheating purposes. But in the 'Modified' version, the time (T2) is less than as compared to the time T2 in the previous process (Dual Current Parameter Method). T2 comes out to be 14seconds. This time T2 causes a deflection of just $1 \mathrm{~mm}$, so the need for stabilizing time is ruled out. In the end, A fast moving motor of $80 \mathrm{rpm}$ is used, which can calibrate the piece in around $3 \mathrm{sec}$, which is 5 times quicker than the previous process. Since this a very fast process, requiring a total time of $18 \mathrm{sec}$, this makes the effect of temperature changes occurring while the calibration process on the MCB negligible, which were more prominent in the previous case as it was a longer process

For an 80rpm motor,

80 rotations::: $1 \mathrm{~min}=60 \mathrm{sec}$

1 rotation::: $60 / 80=0.75 \mathrm{sec}$

For 4.5 rotations (for full calibration process) ::: $0.75 * 4.5=3.37 \mathrm{sec}$

\section{Verification of the process}

Result of both the Calibration procedures wiz. Dual Current Parameter Method and the Single Current Parameter were tested out. Testing was carried out at individual Calibration benches at the Havells [1] laboratory maintaining same temperature conditions. Real Cam application was used to view the real-time result of both the benches, using ten MCBs at a given time. MCBs used were of 16Amp rating and were randomly selected from the given lot. All the $20 \mathrm{MCBs}, 10$ from each procedure were then verified to be calibrated correctly; however, the time taken by Single Current Parameter was found to be $68 \%$ faster than the previous one.

\section{Conclusion}

The Single Current Parameter Method of Calibration gives the same result as the previous method i.e. Dual Current Parameter Method, at the same time taking lesser time and thus increasing the production. The combination of magnetic actuator and vacuum interrupters has enabled maintenance-free for life circuit breakers. Switchgear can now be made more compact, simple and reliable by bolting the circuit breaker into the high voltage compartment. The concepts already developed can be applied to any form of switchgear likely to be used in the Electric Warship

\section{References}

\section{[1] HAVELLS INDIA PVT LTD, BADDI PLANT.}

[2] Xiaoyong, Zhang Xiaoyan, Yao Fang Li Wenhua, Wu, "Research on the reliability test control system's design of the miniature circuit breaker" IEEE International Symposium on Industrial Electronics, 2009. ISIE 2009, 5-8 July 2009, Page(s): 1380 - 1384.

[3] Wolff, H.W," Integration of miniature circuit breakers into distribution networks", Proceedings of the Institution of Electrical Engineers, August 1970, Volume: 117, Issue: 8, Page(s): 1546 - 1560.

[4] YanYan Luo; JianGuo Lu; ZhiGang Li , "Study of reliability test and analysis for miniature circuit breakers", Proceedings of the Forty-Eighth IEEE Holm Conference on Electrical Contacts, 2002, Page(s): 80- 85.

[5] Wolff, H.W., "Design, performance and application of miniature circuit-breakers". Proceedings of the IEE - Part A: Power Engineering, June 1955, Volume: 102 , Issue: 3, Page(s): 364- 373

[6] J Rye, L J Makay, J C Tobias, 'The advantages of fixed circuit breaker switchgear', Fifth International Conference on Trends in Distribution Switchgear.

[7] International Standard IEC 60898-1 and European Standard EN 60898-1 that define the rated current In of a MCB for low voltage distribution applications as the current that the breaker is designed to carry continuously (at an ambient air temperature of $30{ }^{\circ} \mathrm{C}$ ).

\begin{tabular}{|l|l|l|l|l|}
\hline & I1 & T1 & T2 & T3 \\
\hline RATING & Preheating & Justification & Preheating & Calibration \\
\hline
\end{tabular}




\begin{tabular}{|l|l|l|l|l|}
\hline & & & & \\
\hline Units & Amp & Sec & Sec & Sec \\
\hline 6 amp & 16 & 1 & 8 & 10 \\
\hline $10 \mathrm{amp}$ & 25 & 1 & 13 & 16 \\
\hline $16 \mathrm{amp}$ & 38 & 1 & 15 & 18 \\
\hline $20 \mathrm{amp}$ & 52 & 1 & 14 & 17 \\
\hline $25 \mathrm{amp}$ & 63 & 1 & 16 & 19 \\
\hline $32 \mathrm{amp}$ & 80 & 1 & 15 & 18 \\
\hline
\end{tabular}

Table 1. For Single Current Parameter Method

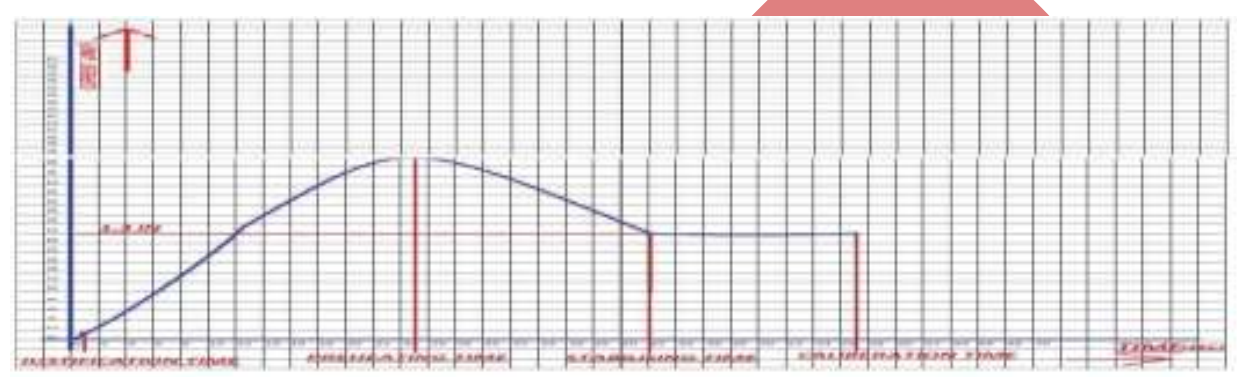

Fig 6 Time Current Characteristics for Dual Current Parameter Method of Calibration

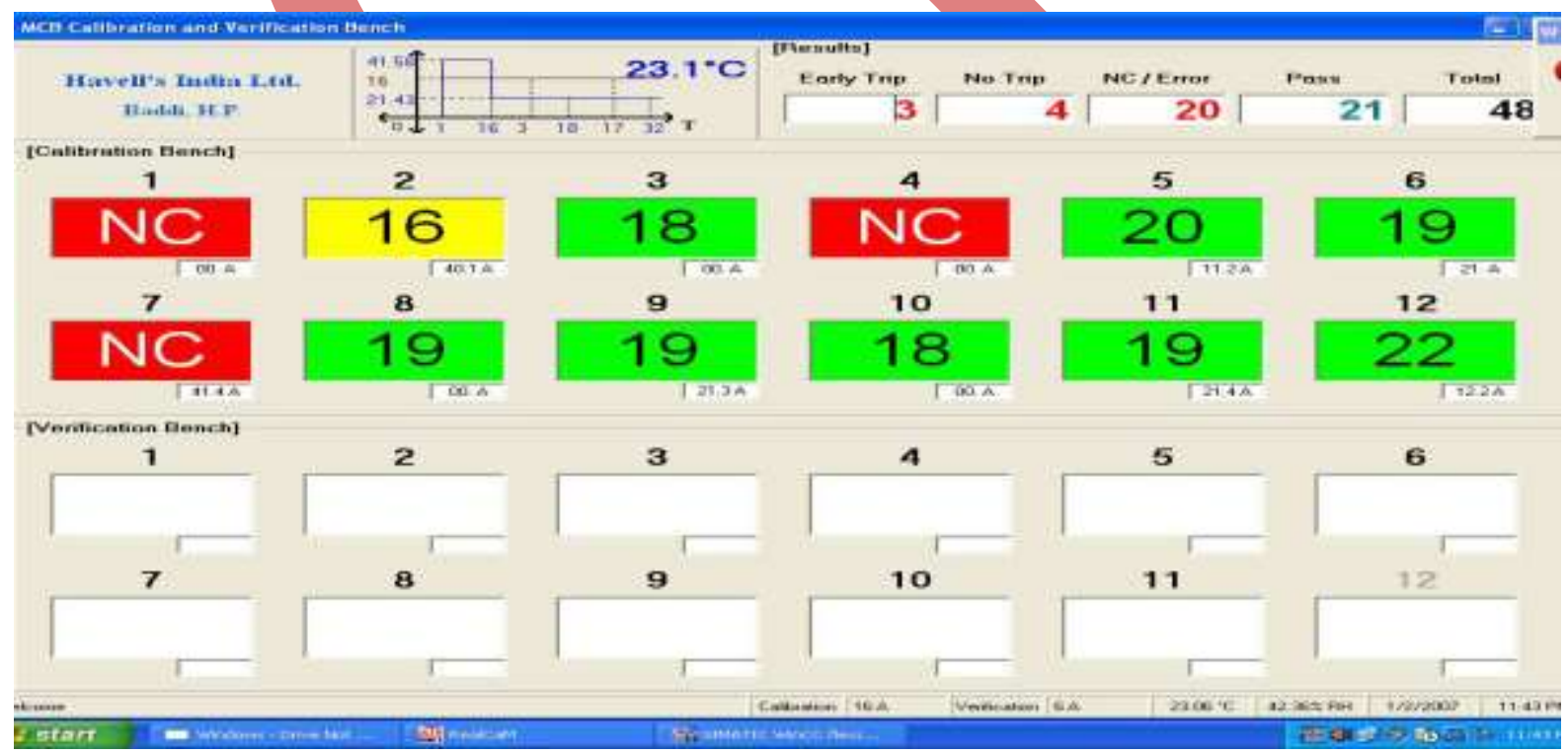

Fig 7 Calibration of 16 Amp MCB using Single Current Parameter using 80rpm motors 